\title{
17-Symmetry and its Generalizations
}

MS-17.01.04 ALGORITHM FOR HIGH DIMENSIONAL POLYTOPE VISUALIZATIONS. By S. S. Im and H. S. Kim*, Dept of Inorganic Materials Engineering, College of Engineering, Chonnam National University, Yongbong-Dong, Buk-Gu, Kwang $\mathrm{J}_{\mathrm{u}, \text { 500-757, Korea. }}$

Many articles deal with studies of symmetry in higher dimensions and with applications to incommensurate structures and to quasicrystals. It is very difficuit to perceive high-dimensional features. Polytope is the simplest symmetrical shape for representing the space. In this presentation, we develop an algorithm to visualize high-dimensional polytopes with computer graphics in personal computers.

Polytopes are composed of vertices and edges. Vertices are determined by intersections of hyper-planes, and edges are obtained by reducing dimensions. Through use of endless loops in the calculation, the dimension of polytopes is restricted only by available computer memory. Several projections, stereoviews and animations are applied to help our perception of higher dimensional space. In the simplest case, hypercubic, this algorithm requires little computing time for the construction of the hypercube. The interesting relations between the vertices and edges of the hypercube will be discussed. We look forward to discussions about symmetry operations in higher dimensions to improve our understanding.

MS-17.01.05 CRYSTALS ON A WEIQI BOARD: PERIODIC TWOCOLOURINGS OF A NET. J. S. Rutherford, Department of Chemistry, University of Transkei, South Africa.

This work concerns the number of distinct structural derivative lattices (commensurate superlattices) that can be constructed by the periodic arrangement of a number of structural motifs, (represented by colours), on a regular basic lattice. In general the method used consists of four steps:

1. The counting of the derivative lattices by index (Rutherford, Acta Cryst., 1992, A48, 500-508).

2. The identification of the colour lattice groups that may occur for a particular index (Harker, Proc. Natl. Acad. Sci. USA, 1978, 75, 5264-5257), and the partition of the total number of derivative lattices for that index by colour lattice group.

3. The application of Polya's Theorem (Polya, Acta Math., 1937, 68, 145-254) to provide the total number of patterns for each colour lattice group and stoicheometry.

4. The elinination of patterns already counted under derivative lattice of lower index.

The simplest case of practical importance, that of two colours only on a two-dimensional net, can be usefully modelled using the black and white stones and reticulated board of the game Weiqi (Japanese: Go), and a number of such examples will be illustrated.

MS-17.01.06, THY IITDUCED REPRESEITATIONS OF THE FOTNT GROUP'S TITA TRE SUBDUCED FTPRESENTATIOUS OF THEIR SUB-

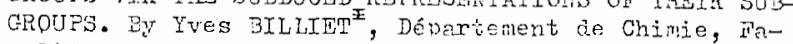
culté des Scinnces, Boite Postale 825, Nimey, Niger.
It is easy to obtain the subduced representations of a point group onto its subgroups. This has been done for all point groups and there exist numerous tables of subduced representations (e.g., Atkins, Child \& Thillips, 1970, Tebles for Group Theory, Oxford, University fress). As an example, here are the representations subduced from the irreducible representations of the point group $\overline{4} 2 \mathrm{~m}$.

\begin{tabular}{rrrrrrrr}
$\overline{4} 2 \mathrm{~m}$ & 1 & ${ }^{2}{ }_{\mathrm{C}}$ & $\mathrm{C}_{\mathrm{a}}$ & $\mathrm{m}$ & 222 & $\mathrm{~mm} 2$ & $\overline{4}$ \\
\hline $\mathrm{A}_{1}$ & $\mathrm{~A}$ & $\mathrm{~A}$ & $\mathrm{~A}$ & $\mathrm{~A}^{\prime}$ & $\mathrm{A}$ & $\mathrm{A}_{1}$ & $\mathrm{~A}$ \\
$\mathrm{~A}_{2}$ & $\mathrm{~A}$ & $\mathrm{~A}$ & $\mathrm{~B}$ & $\mathrm{~A}^{\prime \prime}$ & $\mathrm{B}_{1}$ & $\mathrm{~A}_{2}$ & $\mathrm{~A}$ \\
$\mathrm{~B}_{1}$ & $\mathrm{~A}$ & $\mathrm{~A}$ & $\mathrm{~A}$ & $\mathrm{~A}^{\prime \prime}$ & $\mathrm{A}$ & $\mathrm{A}_{2}$ & $\mathrm{~B}$ \\
$\mathrm{~B}_{2}$ & $\mathrm{~A}$ & $\mathrm{~A}$ & $\mathrm{~B}$ & $\mathrm{~A}^{\prime}$ & $\mathrm{B}_{1}$ & $\mathrm{~A}_{1}$ & $\mathrm{~B}$ \\
$\mathrm{~B}^{\prime}$ & $2 \mathrm{~A}$ & $2 \mathrm{~B}$ & $\mathrm{~A}+\mathrm{B}$ & $\mathrm{A}^{\prime}+\mathrm{A}^{\prime \prime}$ & $\mathrm{B}_{3}+\mathrm{B}_{2}$ & $\mathrm{~B}_{2}+\mathrm{B}_{1}$ & $\mathrm{E}$
\end{tabular}

Consider an irreducible representetion $\pi^{\circ}(G)$ of a group $G$ and an irreancible representation $\rho^{\circ}(\mathrm{H})$ of a subgroup H. Accordine to the Frobenius reciprocity theorem, the number of times $\pi^{\circ}(G)$ is conteined in the induced revresentetion $\rho^{\circ}(4) \uparrow G$ into $G$ is equal to the number of times $\rho^{\circ}(H)$ is continged in the subuced renresentation $\pi^{\circ}(G) \downarrow$ onto $H$. For instance let as determine the representation of $\overline{4} 2 \mathrm{~m}$ induced by the representation $A+3 B$ of the suberoup 2 . From the rrevious teble we see thet ${ }_{2}\left(2_{c}\right)$ appears once in $A_{1}(\overline{4} 2 m) \downarrow 2$, once in $A_{2}(\bar{d} 2 m) \downarrow 2_{c}$, once in $3_{1}(\overline{4} 2 m) \downarrow 2 c$ and once in $B_{2}(\overline{4} 2 m) \downarrow 2$. There fore $A_{1}(\overline{4} 2 m), A_{2}(\overline{4} 2 m)$, $\bar{B}_{1}(\overline{4} 2 \mathrm{~m})$ and $B_{2}(\overline{4} 2 n)$ aprear once in $\Lambda\left(2_{c}\right) \uparrow 42 \mathrm{n}$. In the same wey $\mathbb{E}(\overline{4} 2 \mathrm{~m})$ anpears twice in $B\left(2_{c}\right) \uparrow \bar{A} 2 \mathrm{~m}$ seeing that $B\left(2_{c}\right)$ appeers twice in $\mathbb{C}(\overline{4} 2 \mathrm{~m}) \downarrow 2_{c}$. Thus one has $(A+3 B)\left(2_{C}\right) \uparrow \overline{4} 2 m=A_{1}+A_{2}+B_{1}+B_{2}+6 E$, oving to the eaditivity property of representations. Starting from any representation (in reduced form) of the subgroup $\mathrm{F}$, this mrocess enebles to construct easily the connected induced reiresentation (in reduced form) of the group G. In prectice, it is sufficient to have at one's disposal tables of the representations of the noint grouns induced by the irreducible representetions of their subgroups. As an example, here given these tables in the case of the point groups $\frac{2}{42 m}, 32$ and 23.

$\left.\mathrm{A}(1) \uparrow \overline{4} 2 \mathrm{~m}=\mathrm{A}_{1}+\mathrm{A}_{2}+\mathrm{B}_{1}+\mathrm{B}_{2}+2 \mathrm{E}, \quad \mathrm{A}_{2}\right) \uparrow \overline{4} 2 \mathrm{~m}=\mathrm{A}_{1}+\mathrm{A}_{2}+\mathrm{B}_{1}+\mathrm{B}_{2}$, $B\left(2_{c}\right)+\overline{4} 2 m=2 \mathrm{E}, A\left(2_{2}\right) \uparrow \overline{4} 2 \mathrm{~m}=\mathrm{A}_{1}+\mathrm{B}_{1}+\mathrm{E}$, $B(2 a) \uparrow \overline{4} 2 m=A_{2}+B_{2}+B^{2}, A^{\prime}(m) \uparrow \overline{4} 2 m=A_{1}+B_{2}+E$, $A^{\prime \prime}(m) \uparrow \overline{4} 2 m=A_{2}+B_{1}+E, A(222) \uparrow \overline{4} 2 m=A_{1}+B_{1}$,

$3_{3}(222)+\overline{4} 2 \mathrm{~m}=\mathrm{E}, \mathrm{B}_{1}(222)+\overline{4} 2 \mathrm{~m}=\mathrm{A}_{2}+\mathrm{B}_{2}$, $\mathrm{E}_{2}(222) \uparrow \overline{4} 2 \mathrm{~m}=\mathrm{E}, \mathrm{A}_{1}(\mathrm{~mm} 2) \uparrow \overline{4} 2 \mathrm{~m}=\mathrm{A}_{1}+\mathrm{B}_{2}$, $\bar{B}_{2}(\mathrm{~m} n \mathrm{n} 2)+\overline{4} 2 \mathrm{n}=\mathrm{E}, \mathrm{A}_{2}(\mathrm{~mm} 2)+\overline{4} 2 \mathrm{~m}=\mathrm{A}_{2}+\mathrm{B}_{1}$, $B_{1}(m: n 2) \uparrow \overline{4} 2 m=E, \quad A(\overline{4}) \uparrow \overline{4} 2 m=A_{1}+A_{2}$, $\overline{3}(\overline{4}) \uparrow \pi / T 2 m=B_{1}+B_{2}, 1 / 2 E(\overline{4}) \uparrow \overline{4} 2 m=E_{0}+$ $A(1) \uparrow 32=A_{1}+A_{2}+2 E, A(2) \uparrow 32=A_{1}+E, B(2) \uparrow 32=A_{2}+E$, $A(3)+32=A_{1}+A_{2}, 1 / 2 \mathrm{E}(3)+32=\mathrm{E}_{\text {. }}^{+}$

$A(1) \uparrow 23=A+B+3 T, A(2) \uparrow 23=A+E+R, B(2) \uparrow 23=2 T$, $A(222) \uparrow 23=t+E, B_{3}(222) \uparrow 23=T, B_{1}(222) \uparrow 23=T$, $B_{2}(222) \uparrow 23=\mathrm{T}, A(3) \uparrow 23=\mathrm{A}+\mathrm{T}, 1 / 2 \mathrm{~B}(3) \uparrow 23=1 / 2 \mathrm{~T}+\mathrm{T} .^{+}$ $+E(\overline{4}), E(3)$ are 2-rinensional reducible representations consisting of 2 complex-conjugate l-dimeneional irreducible representetions. 